\title{
Comparison of Volume Retention and Biocompatibility of Acellular Dermal Matrix/Hyaluronic Acid Filler to Autologous Fat Grafts in a Mouse Model
}

\author{
Zhongyang Sun $^{1} \cdot$ Hongyi Zhao ${ }^{1}$
}

Received: 2 July 2020/Accepted: 11 July 2020/Published online: 13 October 2020

(C) Springer Science+Business Media, LLC, part of Springer Nature and International Society of Aesthetic Plastic Surgery 2020

Level of evidence $V$ This journal requires that authors assign a level of evidence to each article. For a full description of these Evidence-Based Medicine ratings, please refer to the Table of Contents or the online Instructions to Authors www.springer.com/00266.

Sir,

We read with great interest the article entitled "Comparison of Volume Retention and Biocompatibility of Acellular Dermal Matrix/Hyaluronic Acid Filler to Autologous Fat Grafts in a Mouse Model" by Kim et al. [1] in Aesthetic Plastic Surgery. In this article, the authors compared the volume retention and biocompatibility of acellular dermal matrix/hyaluronic acid filler (ADM/HA) with fat grafts. The results suggested a favorable prospect of ADM/HA filler as an alternative to fat grafts.

The study was performed using a BALB/c mouse model. Since BALB/c mice have a normal immune system, we should take the impact of immune rejection on graft outcome into consideration.

ADM/HA filler and human fat were injected into either side of the head and tail on the backs of mice, based on the centerline of the forelimbs and hindlimbs. From Fig. 1A, we can see that the grafts were close to the forelimbs and hindlimbs, which are the most locomotive sites. It was reported that muscle mobility can negatively affect fat graft viability [2]. Thus, we think it might be more appropriate to

Hongyi Zhao

17801014026@163.com

1 Department of Plastic Surgery, National Center of Gerontology, Institute of Geriatric Medicine, Beijing Hospital, No. 1, Dahua Street, Dongcheng District, Beijing 100730, People's Republic of China inject on the left and right side of the back, due to the relative immobilization of the recipient sites.

In addition, we suggest that the authors elucidate the process of fat grafting in more detail because different methods of liposuction, processing and reinjection would affect fat viability [3].

\section{Compliance with Ethical Standards}

Conflict of interest The authors declare that they have no conflicts of interest to disclose.

Human and Animal Rights This article does not contain any studies with human participants or animals performed by any of the authors.

Informed Consent For this type of study informed consent is not required.

\section{References}

1. Kim JH, Kim SE, Kim YJ et al (2020) Comparison of volume retention and biocompatibility of acellular dermal matrix/hyaluronic acid filler to autologous fat grafts in a mouse model. Aesthet Plast Surg 44:986-992

2. Shi N, Guo S, Su Y et al (2018) Improvement in the retention rate of transplanted fat in muscle by denervation. Aesthet Surg J 38:1026-1034

3. Jin S, Yang Z, Han X et al (2020) Blood impairs viability of fat grafts and adipose stem cells: importance of washing in fat processing. Aesthet Surg J sjaa170. Online ahead of print

Publisher's Note Springer Nature remains neutral with regard to jurisdictional claims in published maps and institutional affiliations. 